\title{
Partner notification of chlamydia infection in primary care: randomised controlled trial and analysis of resource use
}

Nicola Low, Anne McCarthy, Tracy E Roberts, Mia Huengsberg, Emma Sanford, Jonathan A C Sterne, John Macleod, Chris Salisbury, Karl Pye, Aisha Holloway, Andrea Morcom, Rita Patel, Suzanne M Robinson, Paddy Horner, Pelham M Barton, and Matthias Egger for the Chlamydia Screening Studies (ClaSS) Project Group

\begin{abstract}
Objective To evaluate the effectiveness of a practice nurse led strategy to improve the notification and treatment of partners of people with chlamydia infection.

Design Randomised controlled trial.

Setting 27 general practices in the Bristol and Birmingham areas.

Participants 140 men and women with chlamydia (index cases) diagnosed by screening of a home collected urine sample or vulval swab specimen.

Interventions Partner notification at the general practice immediately after diagnosis by trained practice nurses, with telephone follow up by a health adviser; or referral to a specialist health adviser at a genitourinary medicine clinic. Main outcome measures Primary outcome was the proportion of index cases with at least one treated sexual partner. Specified secondary outcomes included the number of sexual contacts elicited during a sexual history, positive test result for chlamydia six weeks after treatment, and the cost of each strategy in 2003 sterling prices.

Results $65.3 \%$ (47/72) of participants receiving practice nurse led partner notification had at least one partner treated compared with $52.9 \%(39 / 68)$ of those referred to a genitourinary medicine clinic (risk difference 12.4\%, 95\% confidence interval $-1.8 \%$ to $26.5 \%)$. Of 68 participants referred to the clinic, $21(31 \%)$ did not attend. The costs per index case were $£ 32.55$ for the practice nurse led strategy and $£ 32.62$ for the specialist referral strategy.

Conclusion Practice based partner notification by trained nurses with telephone follow up by health advisers is at least as effective as referral to a specialist health adviser at a genitourinary medicine clinic, and costs the same. Trial registration Clinical trials: NCT00112255.
\end{abstract}

\section{Introduction}

A national chlamydia screening programme in England and increasing primary care provision of sexual health care are part of the UK government's strategy for tackling increasing rates of sexually transmitted infections. ${ }^{1}$ Partner notification is essential to the control of sexually transmitted infections, ${ }^{2}$ including chlamydia for which reported diagnoses have increased by $66 \%$ in the past five years. ${ }^{3}$ New strategies for managing chlamydia in non-specialist settings are urgently required: $45 \%$ of cases detected in screening pilot sites were diagnosed in general prac- tice. ${ }^{4}$ Genitourinary medicine clinics are, however, failing to cope with the increasing workload. ${ }^{5}$

Partner notification involves informing the sexual partners of someone with a sexually transmitted infection of the possibility of exposure, offering them diagnosis and treatment, and providing advice about preventing future infection. ${ }^{2}$ In the United Kingdom this is usually done by specialist sexual health advisers in departments of genitourinary medicine. ${ }^{6}$ The effectiveness of partner notification in non-specialist settings in developed countries is not known. ${ }^{7}$ We carried out a randomised controlled trial to compare the effectiveness of, and resources used by, two strategies for managing cases of chlamydia diagnosed in primary care: partner notification by trained practice nurses at the time of diagnosis, with telephone follow up by health advisers; and referral to a specialist health adviser at a genitourinary medicine clinic.

\section{Methods}

The trial was part of the chlamydia screening studies project (ClaSS), a population based study in which men and women, selected at random from the lists of 27 general practices in the Bristol and Birmingham areas, were invited to provide a home collected urine sample or vulval swab specimen, or both, for testing for Chlamydia trachomatis. ${ }^{9}$ People were eligible if they had a positive chlamydia test result and received the result at their general practice (index cases). To increase recruitment, in the last eight months of the study we also asked general practitioners in study practices to refer additional patients diagnosed as having chlamydia infection to the practice nurse for assessment. The nurse provided antibiotic (azithromycin) treatment, explained the trial, and asked for written consent. Those who agreed were randomised individually using computer generated random numbers in permuted blocks, stratified by practice. Allocation was concealed until assignment by using a central computerised telephone system, which the nurse had to telephone to obtain the assignment.

\section{Interventions}

Index cases randomised to the practice arm had partner notification initiated by a practice nurse immediately after diagnosis. Each practice nominated one or two nurses, who received one day's training about sexual history taking, management of chlamydia, and partner notification, including role play with actors who simulated clinical scenarios. Another half day was spent on training in the study procedures, including enrolment and randomisation. A research health adviser visited each prac- 
tice at the start, was available during the trial by telephone or in person, and carried out telephone follow up. The practice nurse strategy included a partner notification interview, which included a sexual history of all sexual contacts in the six months before the chlamydia diagnosis; patient referral (infected people inform contacts themselves) using contact slips for each partner; advice on avoiding sexual intercourse until the partner had completed treatment; and information about being screened for other sexually transmitted infections. Contact slips included details of the study genitourinary medicine clinics, and requested the treatment centre to return the slip to the study centre. Practice nurses did not follow-up index cases.

Participants randomised to referral to a clinic were given details of a research health adviser at each clinic. If the index case did not telephone the clinic within a week the health adviser made up to two attempts to contact them. Health advisers carried out partner notification using standardised protocols for patient referral, provider referral (immediately informing partners on behalf of the patient), or conditional referral (contacting partners if the patient had not done so after an agreed period) and issued contact slips. They also offered patients a consultation for screening for other sexually transmitted infections. Follow up was by telephone.

\section{Outcomes}

The prespecified primary outcome was treatment of sexual contacts, expressed as the proportion of index cases with at least one sexual partner treated and the number of partners treated per case six weeks after randomisation. A researcher not involved in the participant's partner notification did the telephone follow up. Assessment of outcomes was therefore blinded. We defined a partner as having been treated if at telephone follow up the index case said that the partner had been treated, a contact slip was returned to the study centre, or the partner was confirmed to have attended a local genitourinary medicine clinic after the index case received the intervention. Secondary outcomes included the number of partners per index case elicited in the sexual history and the proportion of index cases with a positive chlamydia test result in a urine sample or vulval swab specimen six weeks after randomisation. We also compared the proportion of index cases with all sexual partners treated, as incomplete partner treatment has been shown to be a risk factor for reinfection after screening for chlamydia. ${ }^{10} 11$

\section{Statistical analysis}

We hypothesised that referral to a specialist health adviser for partner notification would be more effective than the practice nurse strategy. ${ }^{8}$ Our sample size calculation assumed that $60 \%$ of index cases referred to the clinic ${ }^{12}$ and $40 \%$ to primary care would have had at least one partner treated. With $80 \%$ power and a significance level of $5 \%$, this would require 107 participants in each arm.

The primary analysis prespecified in the protocol was carried out according to the intent to carry out partner notification. We included all index cases randomised and all sexual partners elicited, either during the partner notification interview or at telephone follow up for index cases who had not attended the clinic (groups a to $c$ in figure). We assumed that the sexual partners of index cases lost to follow up had not been treated. In a further analysis we included only index cases who received partner notification (groups a and b in figure). We estimated the absolute and relative risks of an index case having at least one partner treated after partner notification by a practice nurse compared with referral to a clinic. We also calculated the mean (standard deviation) number of partners treated per case in the two groups and estimated the difference between means using regression models, with robust standard errors to calculate $95 \%$ confidence intervals. In all analyses we took into account clustering at practice level.

\section{Resource use}

We present the costs of each strategy in obtaining the observed outcome from the perspective of the health service as a cost consequence analysis. ${ }^{13}$ Costs were in sterling at 2003 prices. We obtained hourly rates of pay (including employer contributions) and training costs from the chlamydia screening studies project. Practice nurses recorded the total duration of the consultation, which included the time taken to give results and treatment, explain the study, obtain consent, and carry out randomisation followed by partner notification or referral. We used published data on the duration of clinic consultations for partner notification. ${ }^{14}$

We also estimated the time spent by health advisers providing support for partner notification and telephone follow up. Research health advisers recorded the number and duration of phone calls for following-up partner notification outcomes. We estimated that the initial practice visit lasted two hours and that telephone support to practice nurses averaged 0.1 hours per case. We applied these figures to a population similar to that served by Avon primary care trusts (1 million people, five primary care trusts with 150 general practices, and 1800 cases of chlamydia infection diagnosed in general practice in 2004; Wendi Slater, personal communication, 2005).

\section{Results}

Participants were enrolled from March 2001 to October 2002. Thirty six nurses in 25 of 27 practices involved in the chlamydia screening studies project enrolled at least one participant (median 4, range 1-13). Overall, 74\% (140/190) of eligible participants were randomised: 72 to the practice nurse strategy and 68 to referral to a genitourinary medicine clinic (figure). The two groups were well balanced at baseline (table 1). We obtained outcome data on 74\% (104/140) of index cases and 79\% (163/ 206) of contacts, with similar follow up in both arms (figure). Twenty three contact slips were returned.

All 72 participants randomised to the practice nurse strategy had a partner notification interview on the same day. Of the 68 participants referred to the clinic, 21 (31\%) did not attend, including three who were interviewed by the practice nurse. The remainder had a partner notification interview a mean of 13.2 (SD 18.0) days after randomisation. Sexual histories recorded by practice nurses elicited details of 1.7 (SD 1.2) contacts per case compared with 1.4 (SD 1.0) contacts per case elicited from 47 index cases who were randomised to the clinic and had partner notification done (difference $0.3,95 \%$ confidence interval -0.01 to $0.6 ; \mathrm{P}=0.055$ ).

Overall, $45 \%(92 / 206)$ of contacts of 140 index cases were considered treated: $65.3 \%(47 / 72)$ of index cases seen by a practice nurse and $52.9 \%(39 / 68)$ of those referred to the clinic had at least one sexual partner treated (relative risk 1.2, 0.9 to 1.6, absolute difference $12.4 \%,-1.8 \%$ to $26.5 \%, \mathrm{P}=0.087$; table 2 ). In analysis restricted to index cases who had partner notification done, we found no evidence of a difference between the arms (risk difference $7.9 \%,-8.4 \%$ to $24.0 \%$ ). In the clinic arm, similar proportions of contacts were treated for index cases who attended the clinic $(46.9 \%, 30 / 64)$ and those who did not $(39.1 \%$, $9 / 23)$. Half of index cases seen by a practice nurse $(37 / 72$, $51.4 \%$ ) had all their partners treated compared with $30.9 \%$ (21/ 
Primary care

Table 1 Characteristics of trial participants. Values are numbers (percentages) of participants

\begin{tabular}{|c|c|c|}
\hline \multirow[b]{2}{*}{ Characteristic } & \multicolumn{2}{|c|}{ Partner notification method } \\
\hline & Practice nurse $(n=72)$ & $\begin{array}{l}\text { Genitourinary medicine clinic } \\
\qquad(\mathrm{n}=68)\end{array}$ \\
\hline Women & $49(68)$ & $43(63)$ \\
\hline Men & $23(32)$ & $25(37)$ \\
\hline \multicolumn{3}{|l|}{ Age group (years): } \\
\hline $16-19$ & $28(39)$ & $26(38)$ \\
\hline $20-24$ & $37(51)$ & $33(49)$ \\
\hline $25-39$ & $7(10)$ & $9(13)$ \\
\hline \multicolumn{3}{|l|}{ Ethnic group: } \\
\hline White & 57 (79) & $57(84)$ \\
\hline Black Caribbean & $5(7)$ & $2(3)$ \\
\hline Black other & $1(1)$ & $3(4)$ \\
\hline $\begin{array}{l}\text { Indian, Pakistani, or } \\
\text { Bangladeshi }\end{array}$ & 1 (1) & 0 \\
\hline Chinese or other & $1(1)$ & $2(3)$ \\
\hline Missing data & $7(10)$ & $4(6)$ \\
\hline \multicolumn{3}{|l|}{$\begin{array}{l}\text { Deprivation in ward of } \\
\text { residence }^{\star} \text { : }\end{array}$} \\
\hline Low & $28(39)$ & $26(38)$ \\
\hline Medium & $24(33)$ & $24(35)$ \\
\hline High & $19(26)$ & $17(25)$ \\
\hline Missing data & $1(1)$ & $1(1.5)$ \\
\hline
\end{tabular}

${ }^{*}$ Tertiles of Department of Environment, Transport, and Regions index of multiple deprivations, 2000 score.

68 ) of those referred to the clinic (risk difference $20.5 \%, 4.1 \%$ to $36.9 \% ; \mathrm{P}=0.014)$
Table 2 Outcome of partner notification by practice nurses or specialist at genitourinary medicine clinic. Values are numbers (percentages) unless stated otherwise

\begin{tabular}{|c|c|c|c|c|}
\hline Variables & $\begin{array}{l}\text { Practice } \\
\text { nurse }\end{array}$ & $\begin{array}{l}\text { Genitourinary } \\
\text { medicine } \\
\text { clinic }\end{array}$ & $\begin{array}{l}\text { Difference } \\
(95 \% \text { CI })\end{array}$ & $P$ value \\
\hline $\begin{array}{l}\text { No of index cases randomised } \\
\text { (intention to treat) }\end{array}$ & 72 & 68 & - & - \\
\hline Total No of contacts elicited ${ }^{*}$ & 119 & 87 & - & - \\
\hline No of contacts treated: & 53 & 39 & - & - \\
\hline $\begin{array}{l}\text { Cases with at least one contact } \\
\text { treated } \dagger\end{array}$ & $47(65.3)$ & $36(52.9)$ & $\begin{array}{c}12.4 \\
(-1.8 \text { to } 26.5)\end{array}$ & 0.087 \\
\hline $\begin{array}{l}\text { Mean (SD) contacts treated per } \\
\text { case randomised } \dagger\end{array}$ & $0.74(0.6)$ & $0.57(0.6)$ & $\begin{array}{c}0.16 \\
(-0.02 \text { to } 0.34)\end{array}$ & 0.078 \\
\hline Cases with all partners treated $\neq$ & $37(51.4)$ & $21(30.9)$ & $\begin{array}{c}20.5 \\
(4.1 \text { to } 36.9)\end{array}$ & 0.014 \\
\hline $\begin{array}{l}\text { No of index cases interviewed } \\
\text { (receiving treatment) }\end{array}$ & 72 & 47 & - & - \\
\hline $\begin{array}{l}\text { No of contacts elicited at interview } \\
\text { only }\end{array}$ & 119 & 64 & - & - \\
\hline No of contacts treated: & 53 & 30 & - & - \\
\hline $\begin{array}{l}\text { Cases with at least one contact } \\
\text { treated } \dagger\end{array}$ & $47(65.3)$ & $27(57.4)$ & $\begin{array}{c}7.8 \\
(-8.4 \text { to } 24.0) \\
\end{array}$ & 0.343 \\
\hline $\begin{array}{l}\text { Mean (SD) contacts treated per } \\
\text { case interviewed } \dagger\end{array}$ & $0.74(0.6)$ & $0.64(0.6)$ & $\begin{array}{c}0.1 \\
(-0.1 \text { to } 0.3)\end{array}$ & 0.325 \\
\hline Cases with all partners treated $\neq$ & $37(51.4)$ & $17(36.2)$ & $\begin{array}{c}15.2 \\
(-3.3 \text { to } 33.8)\end{array}$ & 0.108 \\
\hline
\end{tabular}

*Number in clinic arm includes 64 elicited at partner notification interview plus 23 partners of 10 participants randomised to clinic who did not attend appointment.

tOutcome prespecified in study protocol.

$\ddagger$ Outcome not prespecified in study protocol.
Patients positive for Chlamydia $(\mathrm{n}=231)$ :

Chlamydia screening studies project $(\mathrm{n}=219)$

General practice $(n=12)$

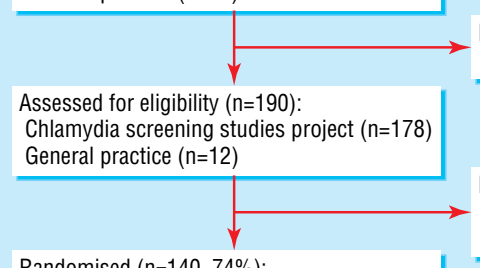

Excluded - did not receive result at practice:

Chlamydia screening studies project $(n=41)$

Excluded - declined randomisation $(n=50)$ : Chlamydia screening studies project $(n=47)$ General practice $(n=3)$

Randomised ( $\mathrm{n}=140,74 \%)$

Chlamydia screening studies project $(n=131)$ General practice $(n=9)$

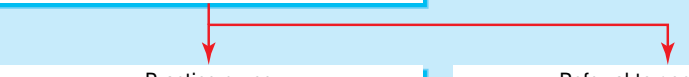

Practice nurse strategy $(n=72)$

Referral to genitourinary medicine clinic strategy $(n=68)$

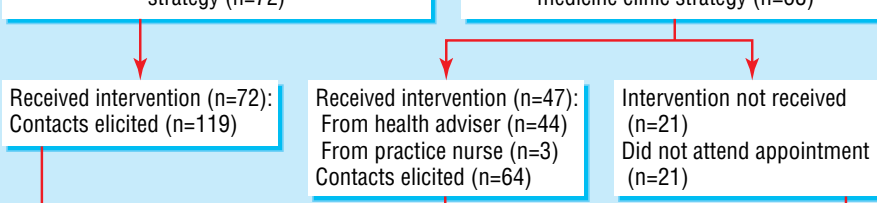

Cases lost to follow up $(n=19)$

Declined follow up $(n=4)$

Not contactable $(n=15)$

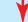

Analysed (a)

Cases ( $\mathrm{n}=72$ )

Contacts $(n=119)$ :

From telephone interview

$(n=74)$

From contact slip or

computer $(\mathrm{n}=22)$

No information $(n=23)$
Analysed (b)

Cases $(n=47)$

Contacts $(n=64)$ :

From telephone interview

$(n=30)$

From contact slip or

computer $(n=14)$

No information $(n=20)$
Cases lost to follow up $(n=17)$

Declined follow up $(n=7)$

Not contactable $(n=10)$

Analysed (c)

Cases $(\mathrm{n}=21)$

Contacts $(n=23)$ :

From telephone interview

$(n=23)$ 
Table 3 Costs of partner notification strategies in primary care

\begin{tabular}{|c|c|c|c|c|c|c|c|}
\hline \multirow[b]{2}{*}{ Variable } & \multicolumn{3}{|c|}{ Practice nurse strategy } & \multicolumn{4}{|c|}{ Genitourinary medicine clinic } \\
\hline & No of participants & Unit cost ${ }^{\star}$ & Total* & $\begin{array}{c}\text { No of } \\
\text { participants }\end{array}$ & Unit cost ${ }^{\star}$ & & Total $^{\star}$ \\
\hline Index case treatment & 72 & 12.00 & 864.00 & 68 & 12.00 & & 816.00 \\
\hline \multicolumn{8}{|l|}{$\begin{array}{l}\text { Mean }(95 \% \mathrm{Cl}) \text { partner } \\
\text { notification advice: }\end{array}$} \\
\hline At general practice & 72 & 11.72 (10.37 to 13.08$) \dagger$ & 843.84 & 68 & 10.86 (9.74 to 11.98$) \ddagger$ & & 738.48 \\
\hline At clinic & - & - & - & 47 & $4.16 \S$ & & 195.52 \\
\hline Partner treatment & 53 & 12.00 & 636.00 & 39 & 12.00 & & 468.00 \\
\hline $\begin{array}{l}\text { Mean }(95 \% \mathrm{Cl}) \text { cost per } \\
\text { index case }\end{array}$ & 72 & - & 32.55 (31.20 to 33.91) & 68 & - & 32.62 & (31.49 to 33.73 ) \\
\hline
\end{tabular}

${ }^{*}$ All costs are United Kingdom sterling in 2003 prices. Cost of nurse £16.80 per hour, health adviser £19.80 per hour, azithromycin assumed to have been used for all index cases and sexual partners.

tCosts include time for giving treatment, explaining study, gaining consent, and undertaking partner notification.

†Costs include time for giving treatment, explaining study, gaining consent, and undertaking partner notification.

$\S C o s t s$ also include time for health adviser consultation for 47 index cases who attended the genitourinary medicine clinic.

Of 73 people (52\%) who returned a specimen six weeks after treatment, one (in the practice arm) had a positive chlamydia test result. This probably represented reinfection because the index case had reported two partners in the past six months, neither of whom had been treated.

\section{Resource use}

Partner notification and treatment undertaken by a practice nurse at the general practice was the dominant option: the cost per index case was $£ 32.55$, and $65.3 \%$ had at least one partner treated. In comparison, referral to the clinic cost $£ 32.62$ per index case, and $52.9 \%$ had at least one partner treated (table 3). For index cases managed at the general practice, nurses took an average of 41.9 minutes (95\% confidence interval 37.0 to 46.7 ) to give the chlamydia test result and treatment, enrol the patient, and carry out a partner notification interview. For those referred to the clinic the average consultation with the practice nurse was 38.8 minutes ( 34.8 to 42.8 ). This included the time taken to give the chlamydia test result and treatment, enrol the patient, and explain the process of referral to the clinic. Index cases who attended the clinic then had a consultation with a health adviser, estimated at 12 minutes (table 3 ). ${ }^{14}$ The one day training course cost an average of $£ 85$ per practice. We estimated locum and travelling costs at $£ 145.79$ per nurse.

Research health advisers spent an average of 4.41 (SD 2.40) minutes on telephone follow up with index cases who could be contacted and 3.73 (SD 1.30) minutes on unanswered phone calls to non-contactable cases. Assuming that, as in this trial, about $75 \%$ of index cases could be contacted at follow up, we estimated that practice visits, telephone support, and follow up for practice nurse led partner notification would require 606 hours of health adviser time for a population the size of Avon.

\section{Discussion}

Practice nurses with appropriate training and support from health advisers to carry out telephone follow up can provide effective immediate partner notification for community diagnosed Chlamydia, and this approach costs the same as standard referral. These results contradicted our hypothesis, that the outcomes of partner notification of chlamydia through referral to a specialist health adviser would be better than those of general practice nurses. This was because about a third of those referred for specialist partner notification did not attend the genitourinary medicine clinic. The costs of the two strategies were similar. Contact slips were not useful for ascertaining contact treatment. Single dose azithromycin seemed to have eradicated chlamydia among those who had follow-up tests six weeks after treatment.

\section{Strengths and weaknesses of study}

The strengths of this study were that it was a multicentre randomised trial, included both sexes, and compared management strategies rather than simply the performance of different professionals. We minimised bias by concealing allocation to study groups, blinding the ascertainment of outcomes, and analysing data according to the intention to carry out partner notification. The outcome was not explained by any imbalance in sexual behaviour between the two arms as the number of partners in the previous 12 months was slightly higher in the referral arm (3.5 v 2.6). One limitation is that low overall uptake of home based screening for chlamydia reduced the number of eligible people. ${ }^{8}$ Confidence intervals for the primary outcome, however, excluded a clinically relevant effect in favour of referral to a health adviser at a genitourinary medicine clinic.

Some features of the research might have influenced the outcomes. For some participants the study clinic was not their nearest clinic: this might have contributed to the $30 \%$ default rate, although this was lower than in some primary care studies. ${ }^{15}$ Giving azithromycin at the practice might have exaggerated the differences between groups if fewer people randomised to the clinic then attended. Conversely, nurses explained to all participants the importance of sexual contacts being treated. This is standard clinical practice, but in a trial setting the differences between groups would diminish if some index cases randomised to referral to the genitourinary clinic acted on this advice and did not attend their appointment. That the proportion of contacts treated in the referral arm was similar whether or not the index case attended the clinic is in keeping with this.

\section{Comparison with other studies}

This is the first randomised trial to have evaluated primary care based partner notification in a developed country. ${ }^{7}$ Contact tracing in UK genitourinary medicine clinics results in the treatment of about 0.61 partners per case seen. ${ }^{12}$ In the English national chlamydia screening programme, $44 \%$ of all contacts identified were treated. ${ }^{16}$ Practice nurse led partner notification compared favourably with these figures $(0.74$ partners per case, $45 \%$ of all partners treated). The combined cost of treatment and management of partners in our study was similar to estimates from the national programme. ${ }^{17}$ Another novel partner notification strategy to enhance patient referral involves index cases delivering antibiotics and sexual health information directly to partners without a clinical consultation, or the partner collecting treatment from a pharmacy. ${ }^{18}$ In a randomised trial in the United States, patient referral and expedited partner therapy together achieved treatment of all partners for an estimated $80 \%$ of index cases. ${ }^{18}$ Similar strategies are being considered in the United 
Kingdom, although contact with the partner (at least by telephone) would be likely to be required for legal reasons.

\section{Meaning of the study}

People diagnosed with chlamydia infection in primary care settings can be managed there by trained staff who are supported by sexual health advisers. Our qualitative research, which will be reported separately, showed that patients also preferred this strategy to clinic referral. Recent research suggests that ensuring that all sexual partners are treated is important for avoiding reinfection. ${ }^{10}{ }^{11}$ Although this outcome was not prespecified, practice based partner notification resulted in more index cases having all partners treated than the referral strategy. This strategy might contribute to improved control of chlamydia in the population if it reduced the duration of infection sufficiently owing to decreased delays in treatment of partners because of the referral process and long clinic waiting times ${ }^{4}$ and a reduction in reinfection from more complete partner treatment. ${ }^{10}{ }^{11}$ We will investigate this, and the cost effectiveness of the strategy, in a separate modelling study. The estimated costs were slightly higher than they would be in practice because trial procedures increased consultation times. These were, however, the same for both study arms. The similarity in practice nurse consultation times between the trial arms suggests that the time taken to explain the referral process to a genitourinary medicine clinic to someone with chlamydia could be spent on taking a sexual history and carrying out partner notification at the practice.

\section{Implications for policy and practice}

Practice nurse led partner notification could be incorporated into the English national chlamydia screening programme, which currently suggests referral to a genitourinary medicine clinic. ${ }^{19}$ This would relieve pressure from overburdened clinics, ${ }^{5}$ which are unlikely to be able to handle the substantial increases in cases of chlamydia generated by a successful national screening programme. Primary care trusts can use our results directly to plan screening because our model of care complied with the core requirements of the national programme ${ }^{19}$ : the central project office received all chlamydia test results, and a health adviser ensured that all patients in a group of practices received treatment, supported practice nurses carrying out partner notification, and followed up the outcomes. This model could be extended to other primary care settings, such as community contraception clinics and NHS walk-in clinics. General practitioners could also be trained. The initial training costs would be discounted over time, and one health adviser could support several primary care trusts.

New interventions to improve health should be shown to be effective before their introduction into routine practice. Free tests for Chlamydia are being introduced to pharmacies in England to increase screening opportunities, ${ }^{20}$ although evidence of effectiveness is lacking. ${ }^{21}{ }^{22}$ In contrast, our study provides high quality evidence that investing in training and support for practice nurse led partner notification in primary care would be an effective use of government resources committed to improving sexual health.

We thank Philippa Matthews, general practitioner principal at Lee Bank Group Practice, Birmingham, who designed, coordinated, and delivered the training package for practice nurses; Anna Graham, who helped deliver the training; the practice nurses who participated in the chlamydia screening studies project and in this trial; and Deborah Hawking for secretarial support throughout the chlamydia screening studies project.

Contributors: NL designed the trial, with input from $\mathrm{MH}, \mathrm{AMcC}, \mathrm{PH}, \mathrm{JM}$, $\mathrm{CS}$, and ME. TER was responsible for the resource use study, which she

\section{What is already known on this topic}

Partner notification is essential for the control of sexually transmitted infections

In the United Kingdom it is usually done by specialist health advisers in genitourinary medicine clinics

The effectiveness of partner notification in primary care settings in unknown

\section{What this study adds}

Trained practice nurses can carry out partner notification for people with chlamydia that is at least as effective as referral to a specialist health adviser

The practice nurse led strategy costs no more than referral to a specialist health adviser

Such a strategy could be incorporated into the national chlamydia screening programme

designed and carried out with help from SMR and PMB. NL and MH chaired the trial working group. AMcC was the ClaSS project manager and supervised the data collection. $\mathrm{AH}$ and $\mathrm{AM}$ coordinated the general practices in Birmingham under the supervision of JM. RP coordinated the general practices in Bristol under the supervision of CS. KP was the lead research health adviser. $\mathrm{PH}$ and $\mathrm{MH}$ supervised clinic based aspects of the trial. ES carried out the statistical analyses under the supervision of NL and JACS. NL wrote the first draft of the paper. AMcC, TR, MH, ES, JACS, JM, $\mathrm{CS}, \mathrm{PH}, \mathrm{KP}$, and ME contributed to the interpretation of the data and made revisions to the draft paper. All authors approved the final draft. NL is guarantor.

Funding: The chlamydia screening studies project was funded through the NHS research and development HTA programme (project No 97/32/31). NL was supported by a NHS career scientist award until 31 March 2005. JM is supported by a NHS career scientist award.

Competing interests: None declared.

Ethical approval: South West multicentre research ethics committee (MREC/00/6/30) and all relevant local research ethics committees.

1 Department of Health. Choosing health:making healthier choices easier. CM 6374. London: Stationery Office, 2004

2 WHO/joint United Nations programme on HIV/AIDS. Sexually transmitted diseases: policies and principles for prevention and care. Geneva: UNAIDS, 1999. [UNAIDS best practice collection]

3 Health Protection Agency. All new episodes seen at GUM clinics: 1999-2003. Country specific tables. London: HPA, 2004.

4 Pimenta JM, Catchpole M, Rogers PA, Hopwood J, Randall S, Mallinson H, et al. Opportunistic screening for genital chlamydial infection. II: prevalence among healthcare attenders, outcome, and evaluation of positive cases. Sex Transm Infect 2003;79:22-7.

5 Adler M. Sexual health. BMJ 2003;327:62.

6 Stokes T, Schober P. A survey of contact tracing practice for sexually transmitted diseases in GUM clinics in England and Wales. Int J STD AIDS 1999;10:17-21.

7 Mathews C, Coetzee N, Zwarenstein M, Lombard C, Guttmacher S, Oxman A, et al. Strategies for partner notification for sexually transmitted diseases. Cochrane Database Syst Rev 2001;(4):CD002843.

8 Low N, McCarthy A, Macleod J, Salisbury C, Horner PJ, Roberts TE, et al. The chlamydia screening studies: rationale and design. Sex Transm Infect 2004;80:342-8.

9 Macleod J, Salisbury C, Low N, McCarthy A, Sterne JA, Holloway A, et al. Coverage and uptake of systematic postal screening for genital Chlamydia trachomatis and uptake of systematic postal screening for genital Chlamydia trachomatis and
prevalence of infection in the United Kingdom general population: cross sectional study. BMJ 2005;330:940.

10 LaMontagne DS, Baster K, Emmett L. Determinants of chlamydia re-infection: the role of partner change and treatment. Abstract TO-405. 16th Biennial meeting of the International Society for Sexually Transmitted Diseases Research. Amsterdam: 10-13 Jul, 2005.

11 Niccolai LM, Ickovics JR, Zeller K, Kershaw TS, Milan S, Lewis JB, et al. Knowledge of sex partner treatment for past bacterial STI and risk of current STI. Sex Transm Infect 2005;81:271-5.

12 Low N, Welch J, Radcliffe K. Developing national outcome standards for the management of gonorrhoea and genital chlamydia in genitourinary medicine clinics. Sex ment of gonorrhoea and

13 Drummond MF, O'Brien BJ, Stoddart G, Torrance G. Methods for the economic evaluation of healthcare programmes. Oxford: Oxford Medical Publications, 2005.

14 Curtis L, Netten A. Units costs of health and social care. Canterbury: University of Kent at Canterbury, Personal Social Services Research Unit, 2003. 


\section{Primary care}

15 Ross JDC, Sutherland S, Coia J. Genital Chlamydia trachomatis infections in primary are. BMJ 1996;313:1192-3

16 LaMontagne DS, Fenton KA, Randall S, Anderson S, Carter P, on behalf of the National Chlamydia Screening Steering Group. Establishing the national chlamydia screening programme in England: results from the first full year of screening. Sex Transm Infect 2004;80:335-41.

17 Adams EJ, LaMontagne DS, Johnston AR, Pimenta JM, Fenton KA, Edmunds WJ. Modelling the healthcare costs of an opportunistic chlamydia screening programme. Sex Transm Infect 2004;80:363-70.

18 Golden MR, Whittington WL, Handsfield HH, Hughes JP, Stamm WE, Hogben M, et al. Effect of expedited treatment of sex partners on recurrent or persistent gonorrhea or chlamydial infection. N Engl J Med 2005;352:676-85.

19 Department of Health. Chlamydia screening programme roll out. Core requirements. Department of Hea

20 Chlamydia-a testing issue. Lancet 2005;365:630.

21 Macleod J, Salisbury C, Low N. Screening for Chlamydia. Lancet 2005;365:1539-40.

22 Van Bergen JE, Postma MJ, Peerbooms PG, Spangenberg AC, Tjen AT, Bindels PJ Effectiveness and cost-effectiveness of a pharmacy-based screening programme for Chlamydia trachomatis in a high-risk health centre population in Amsterdam using mailed home-collected urine samples. Int J STD AIDS 2004;15:797-802. (Accepted 1 November 2005)

doi 10.1136/bmj.38678.405370.7C

Department of Social Medicine, University of Bristol, Bristol BS8 2PR Nicola Low senior lecturer in epidemiology and public health Anne McCarthy research fellow

Emma Sanford research associate

Jonathan A C Sterne reader in medical statistics and epidemiology
Matthias Egger professor of epidemiology

Health Services Management Centre, University of Birmingham, Birmingham B15 2TT

Tracy E Roberts senior lecturer in health economics

Suzanne M Robinson lecturer in health economics

Pelham M Barton lecturer in mathematical modelling

Department of Genitourinary Medicine, Heart of Birmingham Teaching Primary Care Trust, Whittall Street Clinic, Birmingham B4 6DH

Mia Huengsberg consultant

Department of Genitourinary Medicine, United Bristol Healthcare Trust, Milne Centre for Sexual Health, Bristol BS2 8EE

Karl Pye research health adviser

Paddy Horner consultant

Department of General Practice and Primary Care, University of Birmingham John Macleod senior lecturer in primary care

Aisha Holloway research fellow

Andrea Morcom research assistant

Academic Unit of Primary Health Care, University of Bristol, Bristol BS6 6JL Chris Salisbury professor

Rita Patel research assistant

Department of Social and Preventive Medicine, University of Berne, CH-3012, Switzerland

Matthias Egger professor of epidemiology

Correspondence to: Dr N Low, Department of Social and Preventive Medicine, University of Berne, Finkenhubelweg 11, Berne, CH-3012, Switzerland

low@ispm.unibe.ch 